\title{
Designing Computer Aided Design for Malaysia SMEs Food Packaging (PackCAD) using Quality Function Deployment (QFD)
}

\author{
Suziyanti Marjudi \\ Faculty of Information Science \\ and Technology, \\ Universiti Kebangsaan \\ Malaysia, Selangor, Malaysia
}

\author{
Riza Sulaiman \\ Faculty of Information Science \\ and Technology, \\ Universiti Kebangsaan \\ Malaysia, Selangor, Malaysia
}

\author{
Mohd Fahmi Mohamad \\ Amran \\ Faculty of Information Science \\ and Technology, \\ Universiti Kebangsaan \\ Malaysia, Selangor, Malaysia
}

\begin{abstract}
This work explores the application of Quality Function Deployment (QFD) to identify user requirement for development of a new computer-aided design (CAD) system given name as Packaging-Computer Aided Design $($ PackCAD). The paper reviewed literature on QFD, Computer Aided Design (CAD), and Malaysia SMEs Food Packaging. The application of QFD also proposed to obtain feedbacks on the effectiveness of PackCAD respondents on their product packaging design process and user-friendliness.
\end{abstract}

\section{General Terms}

Computer Aided Design, Quality Function Deployment

\section{Keywords}

Data Exchange, Food Packaging, Small Medium Enterprise

\section{INTRODUCTION}

This paper is conducted research on new product development, in particular the tools used to improve the quality and efficiency of the design process. Major work undertaken in the research project was development of an integrated tool. The literature such as Computer Aided Design (CAD) for Food Packaging and Quality Function Deployment (QFD) concept was considered to support the development of the tool. The designs process is articulated in the methodology section. In conclusion the paper highlighted the using of QFD to identify user requirement in PackCAD development.

\section{RELATED WORKS}

\subsection{Computer Aided Design for Malaysia SMEs Food Packaging}

There are various Computer Aided Design (CAD) systems currently available in the market, but mostly are inappropriate. This is due to features that are not applied by users for food packaging design. This is certainly extravagant and incurs a high cost of purchasing the system. Therefore, a CAD system specially designed to fulfil the needs of food packaging SMEs is proposed. It is at par with others in respects of design quality and ease of use [1]. One of the greatest challenges in the development of a CAD system is to make it as very userfriendly, however [16] mention the SMEs have peculiar characteristics such as flexibility, ICT skills and financial resources, which are difficult to be integrated within a structured design network. Designers commonly spend a couple of months to accustom enough with the system, but problems persist when companies transit to other CAD systems or upgrade to later versions [2].

\subsection{Quality Function Deployment}

Quality Function Deployment (QFD) enables the design phase to focus on the key customer requirements, those elements that are defined as being very important to the customer. The design phase is shortened to focus on items that the customer really wants by addressing these elements. By concentrating efforts, less time will be spent on redesign and modifications. The savings have been currently estimated as one-third to one-half of the time taken using traditional means. If a new product took eighteen months from concept to market, by using QFD it could reduce the time to nine to twelve months, with little if any changes to the product once it is in the marketplace [4]. 


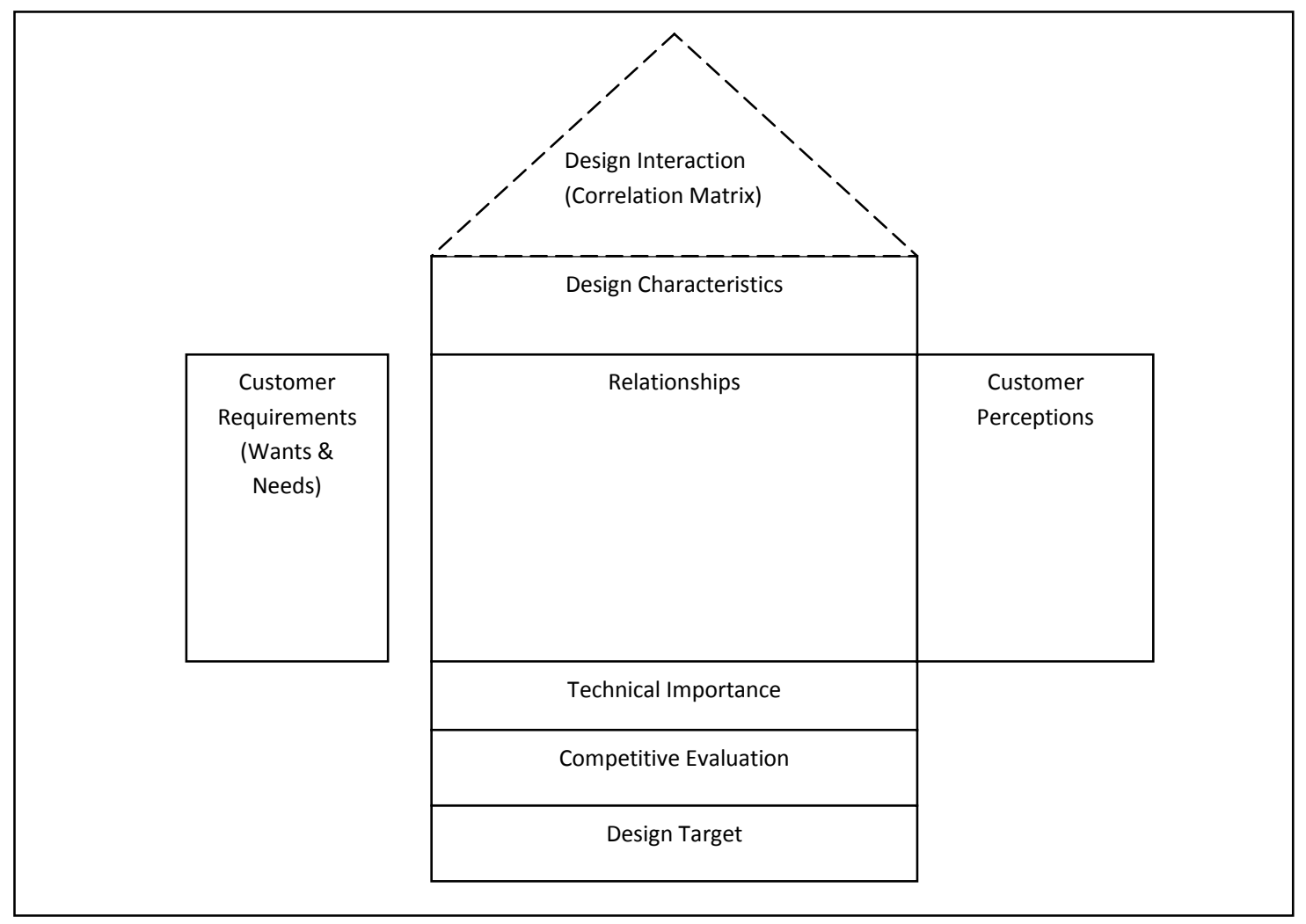

Figure 1.A schematic QFD House of Quality Chart [10]

From the research done by [5], the QFD methodology has proven to be very effective in mapping both the internal and external product requirements to the features contained within a specific architecture. The methodology best suited for expressing a very specific product concept at a specific point. The two dimensional House of Quality is not well suited to relating product requirements, product features, and time all in one chart. In applying the QFD process it was necessary to create two distinct levels of QFD charts, one that mapped product features to product requirements and another that mapped product features to time.

In research paper [6], QFD is applied to outsourcing software development on the basis of analyzing the issues of offshore outsourcing software development and the existing methods of software quality assurance (SQA). Furthermore, a customer satisfaction-oriented model for Outsourcing Software Quality Management (OSQMM) is proposed in this paper. OSQMM stands out by focusing on analyzing and translating the "Voice of Customers". The model is proved to be effective through a case study on the development of an accounting information system. The results from the researcher [7] reemphasize that the design of a product and the manufacturing system to produce it is indeed a process very influenced by the organizational structure and culture that supports it.From finding [10], QFD is a quality-oriented process that can play an important role in the market-driven, total quality control environment. It can be deployed in almost all areas or product development, test and manufacturing processes. QFD is one way to ensure the reliability of the software products. The QFD Process begins by generating or updating the design characteristics from the customer requirement as shown in Figure 1.

\section{RESEARCH METHODOLOGY}

The common methodology applied in systems development is System Development Life Cycle (SDLC). It consists of a sequence of phases where each phase embraces the details of needs and project management. The lifecycle is reversible particular phase can be repeated again if necessary. The main objective of analysis is to review on the techniques available for the exchange or transfer of CAD data. There are potential new method to produce the study such as by [11] approach procedures based on the recovery characteristics of data and [12] using the approach of mapping information between the system design CAD. The methodology applied in researching the combination of SDLC and other methodologies is proposed by [13]. 


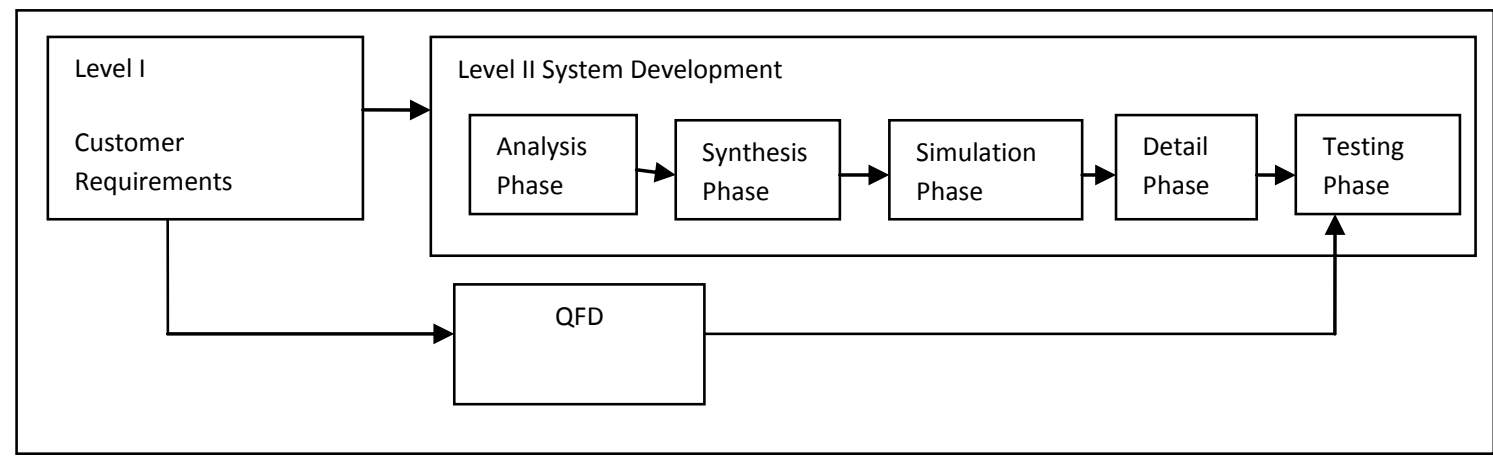

Figure 2. PackCAD development process

\section{Level 1: Initial Analysis}

a) Review the literature of past study: Perform comprehensive literatures systems, current utilizations of $\mathrm{CAD}$ systems in companies and library studies on developers' experiences of CAD

b) Make survey and interview to identify problems and needs: Surveys and interviews are aimed at identifying problems and needs in the use of existing manual systems, infrastructures and designs

Level II: Development of system software PackCAD a) Analysis Phase : Collecting and analyzing the data obtained through interviews with the SMEs and other parties involved. Packaging functions are divided into functional uses and functionalities of the product. A functional use involves protection, distribution and disclosure.

b) Synthesis Phase : The proposed design of food packaging is generated in this phase. A User can choose to design the most appropriate packaging of the food produced. PackCAD is taking into consideration the transfer of data from and/or to other CAD systems is applicable.

c) Simulation Phase : PackCAD system is able to assess whether the design of packaging is made or selected meet the standards or criteria. The concept of packaging design specifications is constructed from a combination of existing or new ideas.

d) Detail Phase: The purpose of packaging is to protect, maintain, market products more effectively, better and safer and to reach customer satisfaction. Thus, this phase is to ensure that the packaging designed meets all its goals. Any changes in this phase involves with other materials, dimensions or printing techniques.

e) Testing Phase: Testing phase uses the Internal Integration $\&$ Test Consumerism.

\section{Result Analysis and Discussion}

A set of questionnaire containing 30 questions divided into three sections was developed. Different question types such as ranking, category, open- ended and scales were used in the questionnaire. The different sections of the questionnaire were i) demographic information of company profile ii) usage of computer aided design system in company iii) for company designer (food packaging). The questionnaire was piloted to a group of $35 \mathrm{SME}$ entrepreneurs to assess its validity before it was distributed. In this study, observations are used for the purposed of design templates food packaging. This research utilized both the quantitative and qualitative research methodology. The instruments used to collect data were questionnaire, interview and observation. [14] The respondents of the study were Malaysia SMEs (Food \& Beverage) company. In November 2010 - June 2011, a total of 320 questionnaires were distributed to company at different states in the country. From the survey has been done strengthen the results of previous studies that were conducted [3], respondents use an outsourcing party such as SIRIM or food packaging manufacturer from government agencies and private sector to design the packaging. Figure 2 show a use of QFD quality tool to evaluate user requirement and testing phase in PackCAD development. QFD determining the needs of end users, special components are identified. In the concept development phase, a designer wants to rapidly create a product's overall shape and user interfaces by making simple sketches, known as 'thumbnail sketches', to evaluate ideas and possibilities [15]. SME users prefer simple and user friendly CAD software. PackCAD propose appropriate template according to the form of frozen food products such as curry puff form a semicircle shape, circle shape form a doughnuts and triangle shape form a samosa. Users must enter values such as the dimensions for the selected form PackCAD will suggest appropriate types of packaging design.

\section{CONCLUSION}

In this paper, we discussed on using of QFD quality tool in identify user requirement on $P a c k C A D$ development to obtain feedbacks on the effectiveness of PackCAD respondents on their product packaging design process and user-friendliness. 
This system is proposed with a special intention and attention to support the design process of packaging food products by Small and Medium Enterprises (SMEs) irrespective of the minimum skills in handling computers. Users are only required to select the pull-place (drag-drop) packaging form made in the template (stored in the data library) PackCAD in standard form or set free to change or resize the form according to the needs of food produced. The design constructed using PackCAD can be easily saved and opened to other CAD system.

\section{ACKNOWLEDGEMENTS}

The research university grant HEJIM-FTSM-FKAB-MTDC101101005 is supported this work. Universiti Selangor (UNISEL) Scholarship Programme also acknowledged with gratitude.

\section{REFERENCES}

[1] S. Marjudi, et al. 2011.The development of CAD system for SME food packaging in Malaysia: A proposal. Proc. Electrical Engineering and Informatics (ICEEI), 2011 International Conference on, pp. 1-4.

[2] S. Marjudi, et al. 2011. A study on CAD systems for food packaging. Proc. Open Systems (ICOS), 2011 IEEE Conference on, pp. 171-174.

[3] A. Yusoff, et al.,2011. Framework of CAD system in food packaging for Malaysian SMEs. Proc. Electrical Engineering and Informatics (ICEEI), 2011 International Conference on, pp. 1-4.

[4] J.L. Bossert. 1991. Quality Function Deployment: A Practitioner's Approach. Book Quality Function Deployment: A Practitioner's Approach, Series Quality Function Deployment: A Practitioner's Approach, ed., Editor ed.^eds., ASQC Quality Press, 1991, pp.

[5] M.S. Leonard and J.B. Bowles. 1994. The application of QFD to computer-system architecture. Proc. Reliability and Maintainability Symposium, 1994. Proceedings., Annual, 1994, pp. 359-365.

[6] X. Wei, et al. 2008. Study of a Customer SatisfactionOriented Model for Outsourcing Software Quality Management Using Quality Function Deployment (QFD). Proc. Wireless Communications, Networking and
Mobile Computing, 2008. WiCOM '08. 4th International Conference on, 2008, pp. 1-5.

[7] J.J. Cristiano, et al. 2001. Key factors in the successful application of quality function deployment (QFD).

[8] W. Keqin, et al. 2009. Application of QFD in Enterprise Requirements-Oriented Quality Management of Higher Education. Proc. Management and Service Science, 2009. MASS '09. International Conference on, 2009, pp. $1-5$.

[9] A. Rahman. 1992. Quality Function Deployment (QFD) in testing. Proc. Automated Testing and Software Solutions, IEE Colloquium on, 1992, pp. 6/1-6/7.

[10] X.F. Liu. 2000. Software quality function deployment. Potentials, IEEE, vol. 19, no. 5, 2000, pp. 14-16.

[11] L. Xiaoxia, et al. 2009. Using procedure recovery approach to exchange feature-based data among heterogeneous CAD systems. in Computer Supported Cooperative Work in Design, 2009. CSCWD 2009. 13th International Conference on, 2009, pp. 716-721.

[12] M. Zeng, et al. 2009. Based on Concurrent Engineering's Board-Type Furniture CAD/CAPP System," in Computer Network and Multimedia Technology, 2009. CNMT 2009. International Symposium on, 2009, pp. 15.

[13] D. Lutters and R. ten Klooster. 2008. Functional requirement specification in the packaging development chain," CIRP Annals - Manufacturing Technology, vol. 57, pp. 145-148, 2008

[14] WAY Wan Yusoff et. al. 2011. The application of Quality Function Deployment (QFD) and Rapid Prototyping (RP) Technology in improving the design of Anti Sleep Driving Alarm. Proceedings of the 2011 International Conference on Industrial Engineering and Operations Management Kuala Lumpur, Malaysia, January $22-24,2011$, pp 68-97.

[15] Cheon, S.-U., Kim, B. C., Mun, D., \& Han, S. 2012. A procedural method to exchange editable $3 \mathrm{D}$ data from a free-hand 2D sketch modeling system into 3D mechanical CAD systems. Computer-Aided Design, 44(2), 123-131.

[16] Germani, M., Mengoni, M., \& Peruzzini, M. 2012. A QFD-based method to support SMEs in benchmarking co-design tools. Computers in Industry, 63(1), 12-29. 